\title{
Could Unnecessary Appendectomies be Avoided Using Neutrophil Count and CRP as a Diagnostic Criterion? Answering an Every-Day Clinical Question
}

\author{
Kripouri Panagiota, Filippou Georgios, Baltopoulos Ioannis, Triga Argyro, Andromanakos Nikolaos, \\ Filippou Dimitrios
}

\begin{abstract}
Background: Despite improvements in diagnostic medicine the accuracy diagnosis of acute appendicitis in several cases is yet difficult. Methods: 76 patients were admitted in the hospital for possible acute appendicitis. White blood cells count (WBC) measurement and C-reactive protein (CRP) were assessed in all patients. Of 76 patients, $62(81 \%)$ were operated on by the diagnosis of acute appendicitis, $2(3 \%)$ were operated on without diagnosis of acute appendicitis and served as a control group, and the remainder 12 patients remained under observation, were finally not operated on and served as controls, too. The 64 patients, who were managed by appendicectomy, were divided in four groups proportionally to the operative findings and histopathological reports. Each group's laboratory tests were investigated for WBC and CRP elevation and sensitivity and specificity of these tests were also estimated .

Results: The sensitivity and specificity of these tests were $69 \%$ and $71 \%$ for neutrophil count and $87 \%$ and $100 \%$ for CRP, respectively. Conclusion: Thereby, patients with normal results in all tests (CRP and WBC) together are unlikely to have acute appendicitis, but it is not absolute and that is why should be evaluated with greater caution.
\end{abstract}

Index Terms-Acute appendicitis, C-reactive protein, neutrophils, white blood cells count

\section{INTRODUCTION}

Despite recent advances in diagnostic methods, the diagnosis of acute appendicitis is quite often a diagnostic problem. Diagnosis of acute appendicitis includes an analogous history, pain at McBurney's point and leukocytosis, has a low

Kripouri Panagiota, Resident in General Surgery; Postgraduate Student, Department of Anatomy and Surgical Anatomy, Medical School, National and Kapodistrian University of Athens, Athens, Greece

Filippou Georgios, MD, Msc, General Surgeon, Department of General and Laparoscopic Surgery, Neo Athinaio MD Hospital, Athens, Greece

Baltopoulos I, MD, Department of Anatomy and Surgical Anatomy, Medical School, National and Kapodestrian University of Athens, Athens, Greece

Triga Argiro, MD, Department of General and Laparoscopic Surgery, Neo Athinaio MD Hospital, Athens, Greece

Andromanakos Nikolaos, MD, Department of Anatomy and Surgical Anatomy, Medical School, National and Kapodistrian University of Athens, Athens, Greece;

Filippou Dimitrios, Chief General Surgeon, Department of Anatomy and Surgical Anatomy, Medical School, National and Kapodestrian University of Athens, Athens, Greece diagnostic accuracy which increases significantly by imaging (ultrasonography, CT or laparoscopy) [1].

Many surgeons have operated on patients with a clinical suspicion of acute appendicitis, even though the preoperative tests (WBC and CRP) were normal. The aim of our work was to study and compare the white blood cells count (especially neutrophils percentage) and CRP values with operative findings and histopathological reports of appendectomies in patients who were operated on for a clinical diagnosis of acute appendicitis. However, the question is, could unnecessary appendectomies be avoided using neutrophil account or/and CRP level as a diagnostic criterion? We feel that in times of economic crisis in health systems this could significantly reduce health-care cost.

\section{PATIENTS AND METHODS}

In this prospective study, 76 patients were admitted in the hospital with abdominal pain particularly in the right iliac fossa. Forty-two (55\%) were male and 34 (45\%) were female. The median age of the patients was 24.5 (range 4-72) years. Of these patients 30 (39\%) were children (range 4-15 years). WBC was measured and CRP was assessed by coagulation technique in all cases. In our laboratory, the normal proportion of neutrophil cells was $40-75 \%$ in white blood count test. CRP was assessed using molecules Latex covered with anti-CRP antibodies. Method is qualitative and it detects CRP quantities over the $6 \mathrm{mg} / \mathrm{dl}$. Of 76 patients, $62(81 \%)$ were operated on by the clinical diagnosis of acute appendicitis, also $2(3 \%)$ were operated on without acute appendicitis and served as a control group and 12 (7 of which children) (16\%) were kept under observation without they take antibiotics. These patients had spontaneous improvement within $24 \mathrm{~h}$ of admission. So, they were not operated on and served as controls, too. The 64 patients, who underwent an appendicectomy, divided in 4 groups proportionally to the surgical findings and histopathological reports. First group: 2 patients with a normal vermiform appendix. Second group: 10 patients with an acute catarrhal appendicitis. Third group: 33 patients with an acute purulent appendicitis. Fourth group: 19 patients with an acute gangrenous appendicitis with or without complication. 
Could Unnecessary Appendectomies be Avoided Using Neutrophil Count and CRP as a Diagnostic Criterion? Answering an Every-Day Clinical Question

Figure 1

NEGATIVE CRP IN RELATIONTO SEVERITY OF ACUTE APPENDICITIS

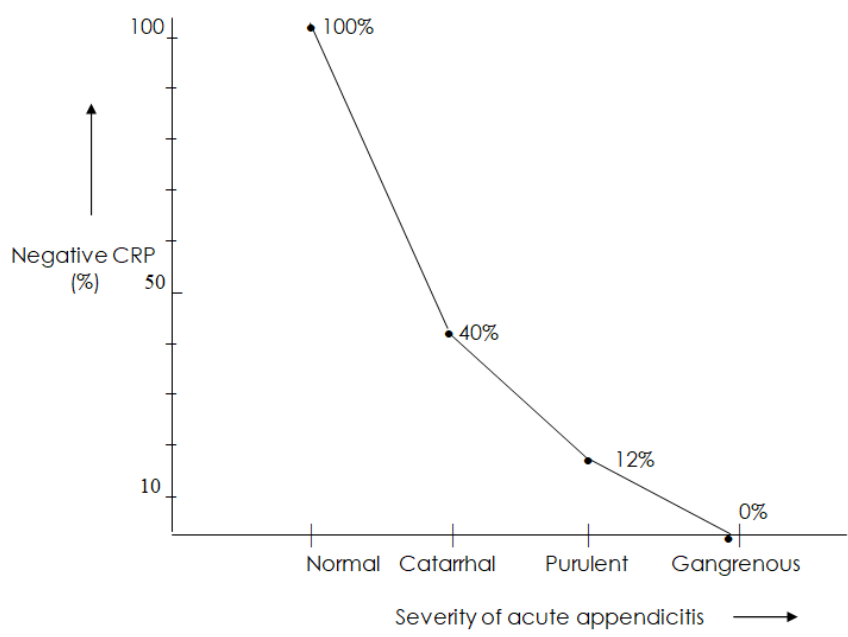

All of the 12 patients, who were not led to the operating theater, had a negative CRP. However, 4 (33\%) of them had an increase of the neutrophil cells. In the 64 operated on patients were showed: (a) In the first group a negative CRP and normal WBC, (b) In the second group a positive CRP $60 \%$ and an increase of neutrophil count $40 \%$, (c) In the third group a positive CRP $88 \%$ and an increase of neutrophil count $70 \%$, and (d) In the fourth group a positive CRP 100\% and an increase of neutrophil count $84 \%$ (Fig. 1). However, in two cases of the third group CRP was negative and neutrophil count was normal. The incidence of acute appendicitis in relation to the age was higher in the second decade of the life (Fig. 2). The acute purulent appendicitis was more frequent in the three first decades of the life, while the acute gangrenous appendicitis showed a higher incidence not only in the two first decades of the life but also in the fifth, sixth and seventh decade of the life, which was all the more severe (Fig. 3).

Figure 2

INCIDENCE OF ACUTE APPENDICITIS IN RELATIONTO AGE

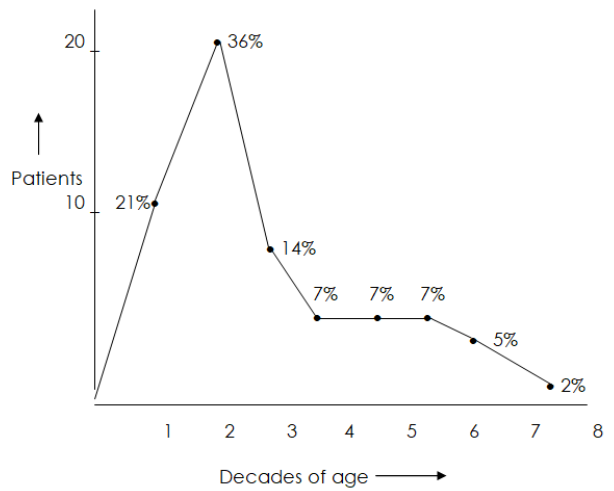

Figure 3

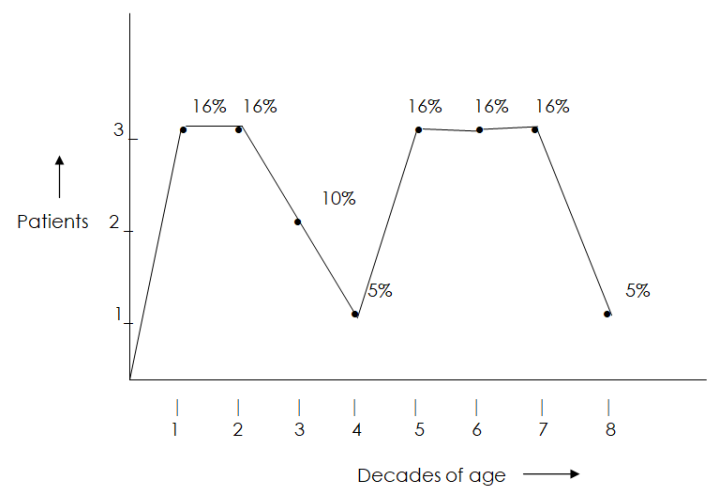

III. DISCUSSION

Acute appendicitis is a very usual surgical disease with a higher incidence in young people [2] as it was also demonstrated in our study. The diagnosis of acute appendicitis and the differential diagnosis of abdominal pain depend on optimal clinical assessment, blood tests and imaging in most cases without a CT scan. [3]

Of 76 patients, who were studied by symptoms of acute appendicitis, $64(84 \%)$ were operated on and $12(16 \%)$ observed without to be operated on. Sixty-two patients with acute appendicitis had CRP level and neutrophil count higher than those of the control group $(\mathrm{p}<0.001)$ unless two cases $(3 \%)$ of acute appendicitis, which had normal blood tests together. In this prospective study, CRP was a better blood test than neutrophil count in all cases of acute appendicitis uncomplicated and complicated, as opposed to the literature that shows that CRP is superior test to leukocyte count only in complicated cases of acute appendicitis [4], [5]. In our study, the sensitivity and specificity of these tests were $87 \%$ and $100 \%$ for CRP and $69 \%$ and $71 \%$ for neutrophil count, respectively. Novel markers for appendicitis have been suggested in the literature however more research is needed to have certain results [6].

\section{CONCLUSIONS}

In conclusion, patients (adult and children) with normal results in all blood tests (CRP, WBC) together are unlikely to have acute appendicitis, but it is not absolute and that is why should be evaluated with greater caution. However, a positive CRP is a valuable diagnostic criterion of acute appendicitis, when other disease (e.g. pelvic inflammatory disease, torsion of ovarian cyst, terminal ileitis, perforated sigmoid diverticulum, pyelonephritis) mimicked acute appendicitis has been excluded.

\section{Conflict of Interest None}

Authors' Contribution

All authors contributed equally to this work.

\section{REFERENCES}

[1] Leeuwenburgh MM, Stockmann HB, Bouma WH, Houdijk AP, Verhagen MF, Vrouenraets B, Cobben LP, Bossuyt PM, Stoker J, Boermeester MA; OPTIMAP Study Group. A simple clinical decision rule to rule out appendicitis in patients 
International Journal of New Technology and Research (IJNTR)

ISSN:2454-4116, Volume-4, Issue-2, February 2018 Pages 114-116

with nondiagnostic ultrasound results. Acad Emerg Med. 2014 May;21(5):488-96.

[2] Noudeh YJ, Sadigh N, Ahmadnia AY.Epidemiologic features, seasonal variations and false positive rate of acute appendicitis in Shahr-e-Rey, Tehran. Int J Surg. 2007 Apr;5(2):95-8.

[3] Yu YR, Shah SR. Can the Diagnosis of Appendicitis Be Made Without a Computed Tomography Scan? Adv Surg. 2017 Sep;51(1):11-28. doi: 10.1016/j.yasu.2017.03.002.

[4] Shogilev DJ, Duus N, Odom SR, Shapiro NI. Diagnosing Appendicitis: Evidence-Based Review of the Diagnostic Approach in 2014. West J Emerg Med. 2014 Nov; 15(7): 859-871.

[5] Glass CC, Rangel SJ. Overview and diagnosis of acute appendicitis in children. Semin Pediatr Surg. 2016 Aug;25(4):198-203.

[6] Acharya A, Markar SR, Ni M, Hanna GB. Biomarkers of acute appendicitis: systematic review and cost-benefit trade-off analysis. Surg Endosc. 2017 Mar;31(3):1022-1031. 